\title{
Association of p16 as Prognostic Factors for Oropharyngeal Cancer: Evaluation of p16 in 1470 Patients for a 16 Year Study in Northeast China
}

\author{
Hong-xue Meng, ${ }^{1}$ Su-sheng Miao, ${ }^{2}$ Kexin Chen, ${ }^{1}$ Hui-ning Li, ${ }^{3,4}$ Guodong Yao, ${ }^{1}$ \\ Jiashi Geng, ${ }^{5}$ Hongmei Wang, ${ }^{1}$ Qing-tao Shi, ${ }^{1}$ Jing He, ${ }^{1}$ Xionghui Mao, ${ }^{2}$ Fang-jia Tong, \\ Lan-Lan Wei, ${ }^{6}$ Ji Sun, ${ }^{2}$ Dongfeng Tan, ${ }^{1}$ Qi You, ${ }^{7}$ Xiaomei Li $\mathbb{D}^{1},{ }^{1}$ and Jing-shu Geng $\mathbb{D}^{1}$ \\ ${ }^{1}$ Department of Pathology, Harbin Medical University Cancer Hospital, Harbin, China \\ ${ }^{2}$ Department of Otolaryngology, Head and Neck Surgery, Harbin Medical University Cancer Hospital, Harbin, China \\ ${ }^{3}$ Department of Pathology, The First Affiliated Hospital of Heilongjiang University of Chinese Medicine, Harbin, China \\ ${ }^{4}$ Department of pathology, Harbin Medical University, Harbin, China \\ ${ }^{5}$ Department of Radiology, Harbin Medical University Cancer Hospital, Harbin, China \\ ${ }^{6}$ Department of Microbiology, Harbin Medical University, Harbin, China \\ ${ }^{7}$ Department of Gastroenterology, Harbin Medical University Cancer Hospital, Harbin, China
}

Correspondence should be addressed to Xiaomei Li; fanliwenqi@163.com and Jing-shu Geng; gengjingshu@yeah.net

Received 28 November 2017; Revised 22 December 2017; Accepted 23 January 2018; Published 17 September 2018

Academic Editor: Tao Huang

Copyright (c) 2018 Hong-xue Meng et al. This is an open access article distributed under the Creative Commons Attribution License, which permits unrestricted use, distribution, and reproduction in any medium, provided the original work is properly cited.

\begin{abstract}
Human papillomavirus (HPV) is an etiological risk factor for oropharyngeal squamous cell carcinomas (OPSCC). Our study investigates the prevalence, prognostic, and clinicopathologic features of HPV-related oropharyngeal cancer in Northeast China and elucidates the involvement of p16 in the tumorigenesis and progression of OPSCC. Specimens from 1470 OPSCC patients collected from 2000 to 2016 were analyzed using the status of HPV by polymerase chain reaction (PCR) and p16 immunohistochemistry. Overexpression of p16 was observed in 81 (5.51\%) of the 1470 cases, and HPV positive was present in 78 cases (5.31\%) of the 1470 cases. HPV positive and p16 overexpression have a good concordance. However, we found that the etiological fraction of HPV in cancers of the OPSCCs was obviously lower in Northeast China than other cohorts previously reported. Interestingly, nearly $89 \%$ of patients with p16 expression were smokers, and nearly $70 \%$ of patients with p16 expression had a history of alcohol. Our study also demonstrates that p16 expression is significantly associated with early stage primary OPSCCs and the patients with p16 expression tend to show better survival following surgery and radiotherapy.
\end{abstract}

\section{Introduction}

Head and neck squamous cell carcinoma (HNSCC) has been defined as the sixth leading cause of cancer in the world [1]. High recurrence rates and nodal metastases always lead to high mortality of HNSCC. Especially, 5-year survival rates of HNSCC patients with cervical lymph node metastases are reduced by approximately $50 \%$ [2]. Conventionally, patients diagnosed with early stage HNSCC would have good prognosis after surgery and adjuvant radiation $[3,4]$.

Before HPV positive as a new risk factor for HNSCC was found, many risk factors had been reported, including tobacco, poor oral hygiene, and alcohol $[5,6]$. Then the prevalence of HPV-related HNSCC, especially oropharyngeal squamous cell carcinomas (OPSCC), was largely observed in many populations in Western Europe, United States, and Australia [3-7]. Nonetheless, the prevalence, prognostic, significance, and correlations of high-risk HPV infection in OPSCCs in China cohort, accounting for $1 / 4$ of the global population, remain blurry. And the precise pathogenesis and clinic pathologic features of HPV-related oropharyngeal cancer in Northeast China are still unclear.

High-risk human papillomavirus (HPV) infection causes the increase of OPSCC [8]. Many studies have shown that 
the prevalence of HPV-related OPSCC has been evaluated to range from 45 to $90 \%$ [3-7]. Moreover, a dominant subtype of HPV16 is thought to represent 90\% of HPV-related OPSCC. $\mathrm{HPV}$-related OPSCC is identified as a unique clinical entity. Patients with HPV associated SCC are expected to have the improved survival. Thus the clinical value of exploring the role of HPV in OPSCC is also beneficial to decrease treatment related side-effect [8].

p16 protein expression has been reported to be related to HPV infection, and p16 may be used as a predictive biomarker for HPV high-risk tumors [9]. p16 as a cyclin-dependent kinase inhibitor played an important role in inhibiting CDK4 and cyclin D1 complex dependent phosphorylation of $\mathrm{Rb}$ (retinoblastoma), as a tumor suppressor protein [10]. Viral oncoproteins E7 is always expressed in HPV-related cancers. Studies had shown an inhibitory effect of E7 on Rb activation by HPV infection [11, 12]. And inactivation of Rb by $\mathrm{HPV}$-expressed E7 induced the transcription of the cyclindependent kinase inhibitor p16 [13]. Importantly, the expression of p16 was a positive indicator for improved survival. Several researches have demonstrated that p16 was a more effective independent prognostic factor for overall survival and progression-free survival than HPV status prediction $[14,15]$. However, whether p16 immunohistochemistry could be used as a strong discriminator of clinical outcome in patients with OPSCC has not been defined. Larger studies are necessary to determine whether p16 can be used as well established prognostic variables, including $\mathrm{T}$ category, depth of invasion, and nodal status of OPSCC.

In our study, we first investigated the prevalence and prognostic and clinicopathologic features of HPV-related oropharyngeal cancer in Northeast China. Furthermore, we observed that p16 expression was significantly associated with early stage primary OPSCCs and that patients with p16 expression tend to show better survival following surgery and radiotherapy. Our results suggest that p16 may be a prognostic factor of OPSCCs in China.

\section{Methods}

2.1. Patients. This study enrolled 1470 patients with pathology-proven oropharyngeal cancer. Patients were recruited from Harbin Medical University Cancer Hospital (Cancer Center for Northeast China, Harbin, China) from January 2000 to February 2016. Tissues were obtained from patients during surgery.

2.2. Ethics Statement. According to the principles of the Declaration of Helsinki, we conducted this research. All participants in this study signed the written informed consent. The study had been approved by the Institutional Ethics Committee of Harbin Medical University Cancer Hospital.

2.3. Clinical Parameters. The clinical data of controls and IgAN patients, including age, history of smoking, gender, history of alcohol, and treatment, were collected.

2.4. Histopathological Diagnosis. All cases were diagnosed and categorized according to the WHO classification.
All slides were reviewed by two pathologists and scored the pathological variables. International Collaboration on Oropharyngeal Cancer Network for Staging (ICON-S) has developed a TNM classification specific to HPV positive oropharyngeal cancer $[16,17]$. We followed the TNM stage from 7th edition of the UICC/AJCC TNM classification: no lymph nodes as ICON-S N0; ipsilateral lymph nodes as ICON-S N1; bilateral or contralateral lymph nodes as ICON$\mathrm{S}$ N2; lymph nodes larger than $6 \mathrm{~cm}$ as ICON-S N3, which resembles the $\mathrm{N}$ classification of nasopharyngeal carcinoma except the lack of a lower neck lymph node variable. The proposed ICON-S classification is as follows: stage I is T1-T2N0$\mathrm{N} 1$, stage II is T1-T2N2 or T3N0-N2, and stage III is T4 or N3. Metastatic disease (M1) is classified as ICON-S stage IV.

2.5. Antibodies and Immunohistochemistry (IHC). Formalinfixed samples and paraffin-embedded sections ( $4 \mu \mathrm{m}$ thick) were first blocked with $1 \% \mathrm{H}_{2} \mathrm{O}_{2}$. Then the samples were treated by antigen retrieval in trypsin for $30 \mathrm{~min}$ at $37^{\circ} \mathrm{C}$, followed by immersion in citrate buffer ( $\mathrm{pH}$ 6.0; Mitsubishi Chemical Medience, Tokyo, Japan) for $20 \mathrm{~min}$ at $120^{\circ} \mathrm{C}$ in an autoclave. Protein Blocking Agent (Streptavidin-Biotin Universal Detection System; Beckman Coulter, Marseille, France) was used to block the sections. And then the sections were incubated with the following primary antibodies overnight at $4^{\circ} \mathrm{C}$ : rabbit anti-human P16 (1:100, INK4a, IgG, Zhongshan, China). After that, sections were incubated with secondary antibodies from the Streptavidin-Biotin Universal Detection System (Beckman Coulter) and visualized by DAB. The negative controls were specific isotype control antibodies and phosphate-buffered saline (PBS; omitting primary antibodies).

For calculating the p16 [INK4a] expression, nuclear and cytoplasmic positivity were identified as positive reactions and were scored semiquantitatively as described by previous study [15]: negative score was $<1 \%$ of positive cells; sporadic score was that isolated cells were positive but $<5 \%$; focal score was small cell clusters but $<80 \%$ of positive cells; and diffuse score was $>80 \%$ of positive cells. Positive cells with p16 expression were defined as strong and diffuse nuclear and cytoplasmic staining in at least 80 percent or more of the tumor cells.

2.6. DNA Extraction and PCR Analysis. Total DNA was extracted and purified from formalin-fixed, paraffinembedded tissues by DNeasy Micro kit (Qiagen, Hilden, Germany). The resulting DNA was amplified for 35 cycles by PCR. The forward and reverse primers were listed as follows: $\beta$-globin $5^{\prime}$-GAA GAG CCA AGG ACA GGT AC- $3^{\prime}$ (forward) and $5^{\prime}$-CAA CTT CAT CCA CGT TCA CC- $3^{\prime}$ (reverse); HPV $5^{\prime}$-CGT CCM ARR GGA WAC TGA TC-3' (forward) and $5^{\prime}$-GCM CAG GGW CAT AAY AAT GG-3' (reverse).

2.7. Statistical Analysis. Student's $t$-test was performed to estimate the significant difference between HPV positive OPSCC patients and HPV negative OPSCC patients. We also analyzed the correlation among clinical presentation, HPV state, and P16 by Spearman's correlation analysis and 
TABle 1: Profiles and clinical parameters of patients.

\begin{tabular}{|c|c|c|}
\hline \multicolumn{3}{|c|}{ Clinicopathological findings } \\
\hline Variable & $n$ & $\%$ \\
\hline \multicolumn{3}{|l|}{ Age at diagnosis, years } \\
\hline$\leqslant 45$ & 85 & 5.78 \\
\hline $46-55$ & 469 & 31.9 \\
\hline $56-65$ & 624 & 42.45 \\
\hline$\geqslant 66$ & 292 & 19.86 \\
\hline Mean (SD) & $58.24 \pm 6.64$ & \\
\hline Age range & $31-86$ & \\
\hline \multicolumn{3}{|l|}{ Sex } \\
\hline Male & 1167 & 79.39 \\
\hline Female & 303 & 20.61 \\
\hline \multicolumn{3}{|l|}{ History of smoking } \\
\hline Yes (current/former) & 1296 & 88.16 \\
\hline No (never) & 174 & 11.84 \\
\hline \multicolumn{3}{|l|}{ History of alcohol } \\
\hline Yes (current/former) & 890 & 60.54 \\
\hline No (never) & 580 & 39.46 \\
\hline \multicolumn{3}{|l|}{ Treatment } \\
\hline Surgery alone & 1257 & 85.51 \\
\hline Surgery + radiotherapy & 205 & 13.95 \\
\hline Surgery + chemoradiotherapy & 5 & 0.34 \\
\hline $\begin{array}{l}\text { Surgery }+ \text { radiotherapy }+ \\
\text { chemoradiotherapy }\end{array}$ & 3 & 0.2 \\
\hline \multicolumn{3}{|l|}{ Event after initial CRT } \\
\hline Residual tumor (PD, SD, and PR) & 286 & 19.46 \\
\hline CR followed by recurrence/metastasis & 441 & 30 \\
\hline Durable CR & 743 & 50.54 \\
\hline
\end{tabular}

Pearson's correlation analysis (SAS Institute Inc., Cary, NC, USA). $P$ values less than 0.05 were considered as significant differences.

\section{Results}

3.1. Clinical and Pathological Parameters. The clinicopathological characters of the 1470 cases of OPSCC were represented in Table 1. Most of patients were males ( $n=1167$; $79.39 \%)$ and smokers $(n=1296 ; 88.16 \%)$. About sixty percent $(60.54 \%)$ of 890 patients were alcohol consumers. Among all these patients, patients with surgery only were 1257 cases, patients with surgery followed by radiotherapy were 205 cases, patients with surgery followed by chemotherapy were 5 cases, and patients with surgery followed by radiotherapy and chemotherapy were 3 cases. All of the patients had available follow-up information. 286 patients $(19.46 \%)$ presented with residual disease, but 1184 patients $(80.54 \%)$ initially obtained a complete response (CR) after finishing the initial CRT. $62.75 \%(743 / 1184)$ of patients maintained the CR during follow-up; however, 37.24\% (441/1184) of patients subsequently showed recurrence or metastasis.
TABLE 2: Histological diagnosis of patients.

\begin{tabular}{|c|c|c|}
\hline Histological diagnosis & $n$ & $\%$ \\
\hline \multicolumn{3}{|l|}{ Squamous cell carcinoma } \\
\hline SCC NOS/conventional nonkeratinizing & 174 & 11.84 \\
\hline Conventional keratinizing & 1271 & 86.46 \\
\hline Conventional exophytic keratinizing & 14 & 0.95 \\
\hline Basaloid/papillary & 2 & 0.14 \\
\hline Verrucous & 1 & 0.07 \\
\hline Sarcomatoid & 4 & 0.27 \\
\hline Undifferentiated carcinoma & 2 & 0.14 \\
\hline Adenosquamous carcinoma & 2 & 0.14 \\
\hline \multicolumn{3}{|l|}{ Differentiation } \\
\hline Well & 467 & 31.77 \\
\hline Moderate & 818 & 55.65 \\
\hline Poor & 185 & 12.59 \\
\hline Lymphovascular invasion & 615 & 41.83 \\
\hline Perineural invasion & 441 & 30 \\
\hline Extracapsular spread & 244 & 16.6 \\
\hline Bone invasion & 148 & 10.07 \\
\hline \multicolumn{3}{|l|}{ Pathological T category } \\
\hline $\mathrm{T} 1$ & 182 & 12.38 \\
\hline $\mathrm{T} 2$ & 1213 & 82.52 \\
\hline T3 & 61 & 4.15 \\
\hline $\mathrm{T} 4$ & 14 & 0.95 \\
\hline \multicolumn{3}{|l|}{ Pathological N category } \\
\hline N0 & 855 & 58.16 \\
\hline N1 & 422 & 28.71 \\
\hline $\mathrm{N} 2 \mathrm{a}$ & 119 & 8.1 \\
\hline $\mathrm{N} 2 \mathrm{~b}$ & 57 & 3.88 \\
\hline $\mathrm{N} 2 \mathrm{c}$ & 17 & 1.16 \\
\hline N3 & 0 & \\
\hline \multicolumn{3}{|l|}{ Clinical stage } \\
\hline $\mathrm{I} / \mathrm{II}$ & 1338 & 91.02 \\
\hline III/IV & 132 & 8.98 \\
\hline
\end{tabular}

According to the histologic typing, 174 (11.84\%) of the 1470 cases were SCC NOS/conventional nonkeratinizing and $1271(86.46 \%)$ cases were conventional keratinizing. For the differentiation, 467 (31.77\%) cases were well, 818 (55.65\%) cases were moderate, and $185(12.95 \%)$ cases were poor. Most cases $(615,41.83 \%)$ have lymphovascular invasion. For TNM stage statistic, 1395 patients had low-T stage (T1/T2) OPSCC tumors and 75 patients had high-T stage (T3/T4) OPSCC tumors. Moreover, patients with clinically positive lymph node metastasis $(\mathrm{N}+)$ were 615 (41.84\%). For clinical stage statistic, patients with low clinical stage (I/II) were 91.02\% (1338/1470) and high clinical stage (III/IV) were $8.98 \%$ (132/1470) (Table 2).

3.2. The Relationship between p16 Protein Overexpression and HPV Status. 5.51\% (81/1470) of OPSCC samples were detected to p16 overexpression by immunohistochemistry (Figure 1). HPV was positive in 78 cases $(5.31 \%)$ of the 1470 cases by PCR (Figure 2). 


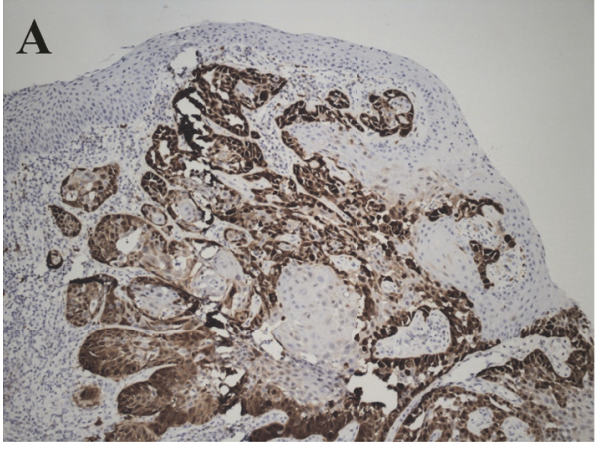

(a)

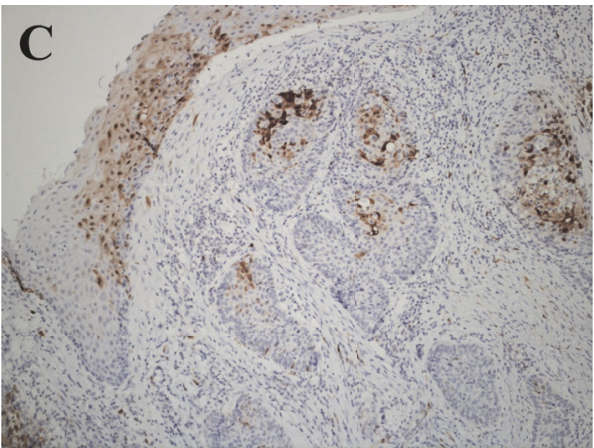

(c)

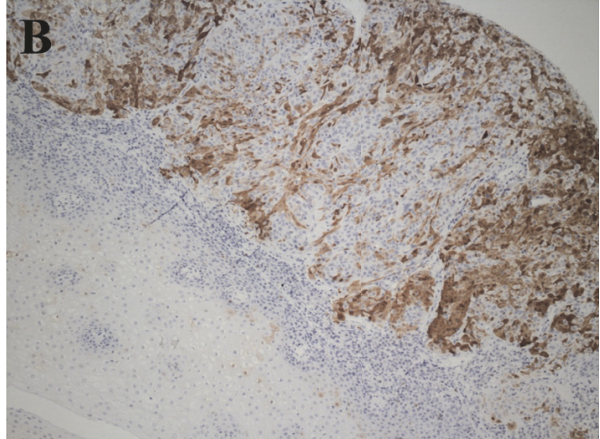

(b)

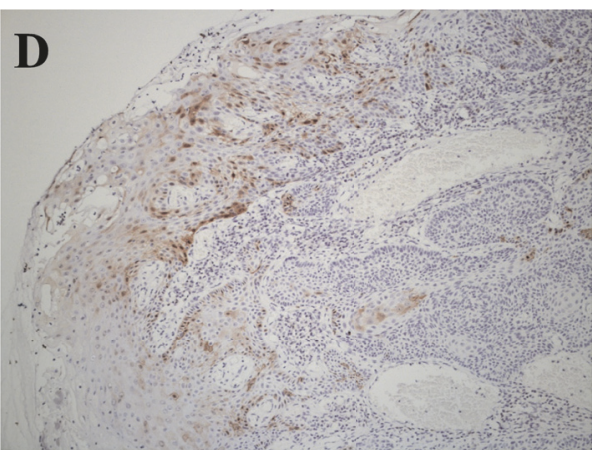

(d)

FIGURE 1: Expression of P16-positive cells in oropharyngeal cancer. Immunohistochemical analysis was used to show the expression of P16positive cells in oropharyngeal cancer (magnification: $\times 400$ ); nuclear and cytoplasmic positivity were classified as positive reactions and were scored as (a) diffuse ( $>80 \%$ of the cells were stained); (b) focal (small cell clusters, but $<80 \%$ of the cells were positive); (c) sporadic (isolated cells were positive but $<5 \%)$; (d) negative $(<1 \%$ of cells were positive).

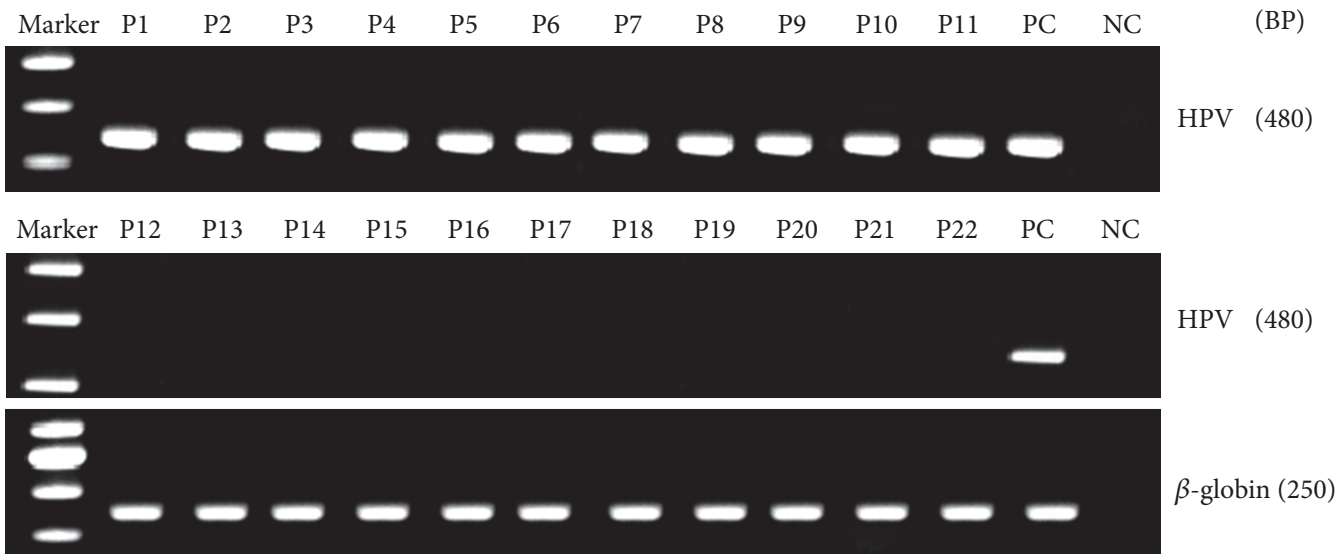

FIGURE 2: HPV DNA-PCR of oropharyngeal cancer. Using PCR, HPV state was detected in Oropharyngeal cancer patients.

Good concordance between HPV positive status and p16 overexpression was established, which was with high sensitivity (100\%) and high specificity (96\%; Table 3). Consistently, we found that HPV status was significantly more frequently present among the young (age: 46-55) $(P<0.01)$, males $(P<$ $0.05)$, conventional keratinizing type $(P<0.01)$, moderate and poor differentiation $(P<0.05)$, low T stage $(P<0.05)$, lymph node metastasis $(P<0.05)$, high clinical stage $(P<$ $0.05)$, and p16 overexpression $(P<0.01)$ cases (Tables 3 and 4). Specifically, HPV and p16 positive patients usually maintained the CR more during follow-up. Our results also indicated that p16 expression may be a prognostic marker with an improved response to both radiation therapy and chemotherapy.

3.3. p16 Expression Was Significantly Associated with Improved Survival. Among all 1470 cases, positive p16 expression was linked with markedly improved overall survival (OS, 


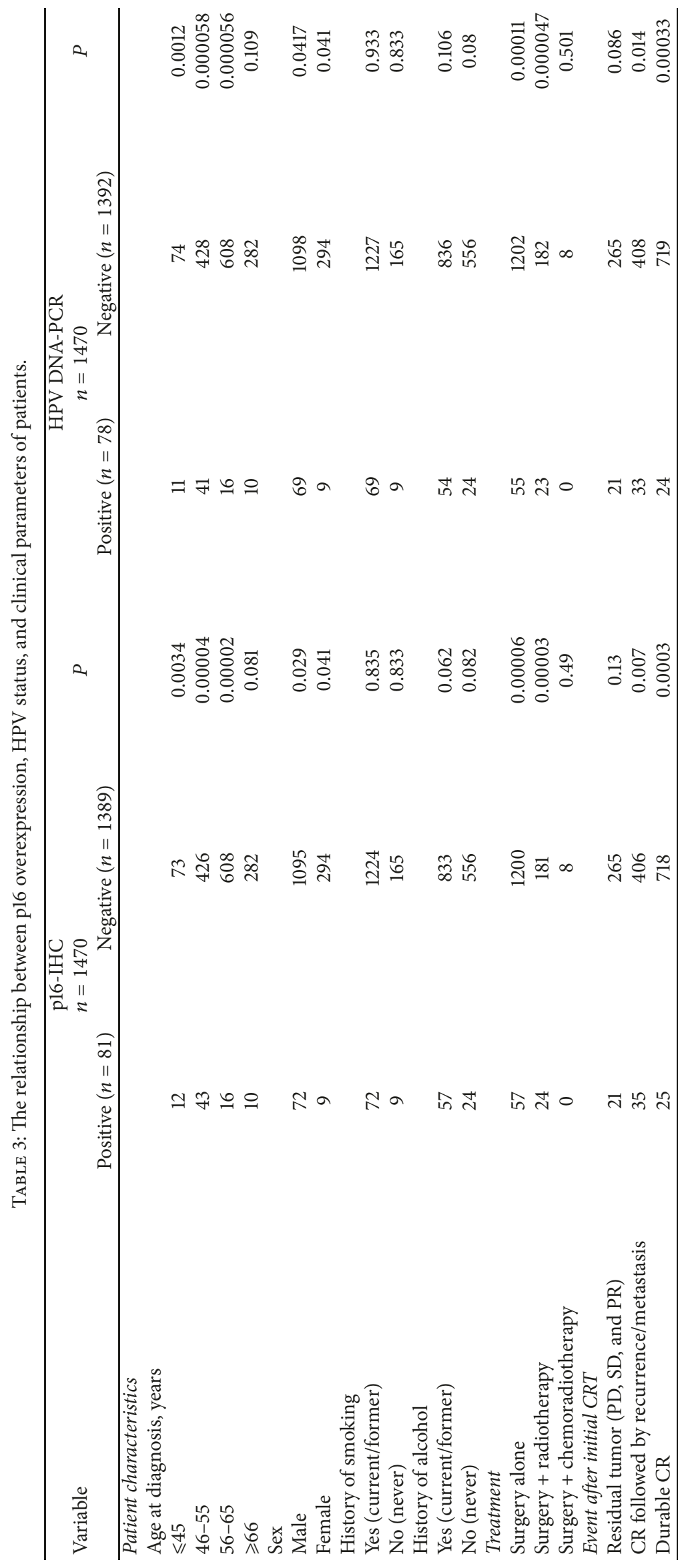




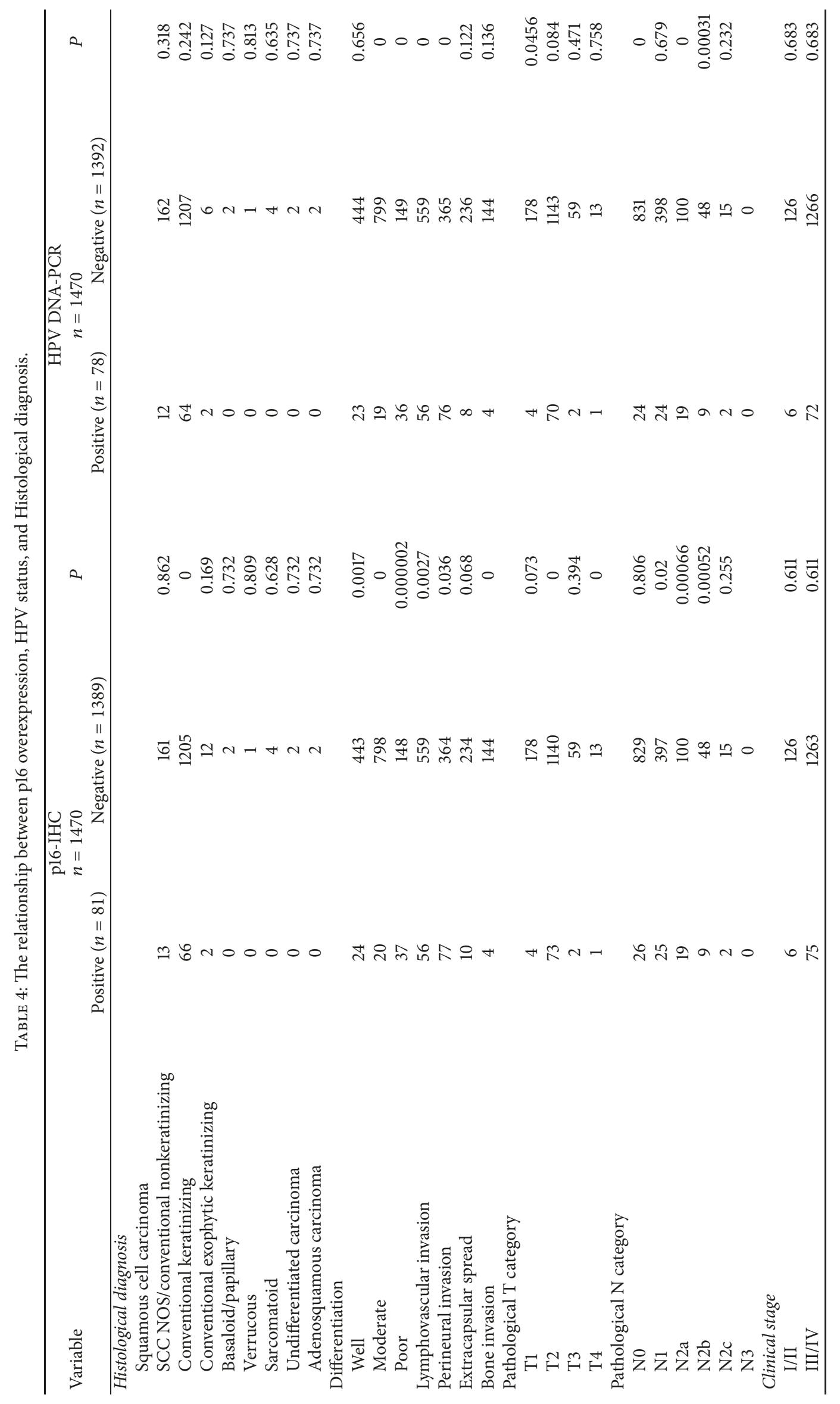


TABLE 5: Association between p16 status and survival.

\begin{tabular}{|c|c|c|c|}
\hline & Univariable ( $p$ value) & $\begin{array}{c}\text { Multivariable, } \mathrm{HR}(95 \% \mathrm{CI}) \\
P \text { value } \\
\end{array}$ & $\begin{array}{l}\text { In patients receiving radiotherapy } \\
(P \text { value })\end{array}$ \\
\hline Disease-specific & $0.03^{\mathrm{a}}$ & $\begin{array}{c}0.19(0.02-1.38) \\
0.100^{\mathrm{b}}\end{array}$ & $0.14^{\mathrm{a}}$ \\
\hline Disease-free & $0.14^{\mathrm{a}}$ & $\begin{array}{c}0.67(0.32-1.36) \\
0.266\end{array}$ & $0.014^{\mathrm{a}}$ \\
\hline Overall survival & $0.05^{\mathrm{a}}$ & $\begin{array}{c}0.44(0.15-1.23) \\
0.118\end{array}$ & $0.038^{\mathrm{a}}$ \\
\hline
\end{tabular}

${ }^{\mathrm{a}}$ Log rank test $P$ value; ${ }^{\mathrm{b}}$ adjusting for effect of depth of invasion alone.

$P=0.05)$, but this result was not significant in multivariate analysis (Table 5).

\section{Discussion}

This study including 1470 patients over a period of 16 years has a few inherent biases typical of a retrospective cohort. The principles of surgical management and patient selection for radiotherapy have essentially remained the same during this period, although there has been increasing use of adjuvant chemotherapy and highly conformal radiation techniques. Consequently, we have used HPV status detection as the gold standard to evaluate the potential clinical value of other prognostic markers for HPV-related OPSCC.

The percentage of HPV positive OPSCC in northeast Chinese patients calculated by our research was substantially lower than that published by recent meta-analysis data in oropharyngeal cancers [18]. The percentage of HPV positive OPSCC was $11.7 \%$ in an Eastern Chinese population and $21.7 \%$ in Southern Chinese patients as previously reported $[19,20]$. The discrepancy may be related to the differences in the geographic origin of patients, heterogeneous laboratory procedures, or different methods used to detect HPV status.

We also found that HPV status was significantly correlated to sex and age of patients. About sex, HPV positive estimates were substantially higher in men than in women in our cohort, different from European cohort in other studies [3-7]. Additionally, we also found that HPV positive estimates were substantially higher in $46-55$ ages. Finally, the incidence of tobacco smoking was $88.16 \%(n=1296)$ in our cohort and nearly $89 \%$ of patients with p16 expression were smokers. Sixty $(60.54 \%)$ of 890 patients were alcohol consumers, and nearly $70 \%$ of patients with p16 expression had a history of alcohol. The carcinogenic effects of smoking and alcohol mediated through p53 mutations are notable.

In our results, we observed that OPSCC patients with p16 overexpression had significantly longer disease-specific survival than p16 negative patients following surgery as well as postoperative adjuvant radiotherapy, which was consistence with published data about the potential prognostic maker of p16 in oropharyngeal cancer. It suggested that p16 may be a biomarker for predicting the prognosis of OPSCC patients in China.

HPV and p16 as biomarkers or therapeutic targets in the treatment of HNSCC have the growing consensus of the importance [21]. In our study, p16 positive patients had significantly longer disease-specific survival on univariable analysis, which was essentially equivalent to that published by previous reports [22-24]. However, p16 expression was not only an independent predictor of survival on multivariable analysis. As discussed above, it may be reasonable to assume that p16 expression may also mediate survival of OPSCC patients by controlling the proliferative capacity and invasive potential of the primary tumor.

\section{Conclusion}

Our study demonstrates that p16 expression is significantly associated with early stage primary OPSCCs and that patients with p16 expression tend to show better survival following surgery and radiotherapy. p16 expression, as well as HPV status, may be a prognostic maker of OPSCCs in China. Furthermore, the etiological fraction of HPV in cancers of the OPSCCs is substantially lower in Northeast China than that in United States and Western Europe. Thus, the real prevalence of HPV in OPSCCs is still the future burden. Further researches will define the more detailed mechanisms underlying HPV involvement in OPSCCs.

\section{Conflicts of Interest}

All authors have read the journal's policy on disclosure of potential conflicts of interest declare that they have no conflicts of interest.

\section{Authors' Contributions}

Hong-xue Meng, Su-sheng Miao, and Kexin Chen contributed equally to this study.

\section{Acknowledgments}

This work was supported National Nature Science Foundation of China (81600539, 81372785, 81400443, and 81372178), Natural Science Foundation of Heilongjiang Province of China (QC2012C041, LC2016038), Foundation of Heilongjiang administration of Traditional Chinese Medicine (Huining Li, ZHY16-032), Chinese Postdoctoral Science Foundation (2015M581472), Special Financial Grant from the China Postdoctoral Science Foundation (2016T90310), Postdoctoral Science Foundation of Heilongjiang Province of China (LBH-Z16101, LBH-TZ0616), Heilongjiang Human 
Resources and Social Security Bureau (Hong-xue Meng), Harbin Special Fund Project for Science and Technology Innovation (2016RAQXJ203), Foundation for Liver and Gall Group of Nn10 Fund Project of Harbin Medical University Cancer Hospital, and Youth Elite Training Foundation of Harbin Medical University Cancer Hospital (JY2016-06).

\section{References}

[1] W. L. Wong, J. Dunn, and H. Mehanna, “The role of PET CT in the management of advanced nodal head neck cancer post chemoradiotherapy," Translational Cancer Research, vol. 5, pp. S932-S932, 2016.

[2] S. Z. Liu, D. P. Zandberg, L. M. Schumaker, J. C. Papadimitriou, and K. J. Cullen, "Correlation of p16 expression and HPV type with survival in oropharyngeal squamous cell cancer," Oral Oncology, vol. 51, no. 9, article no. 3263, pp. 862-869, 2015.

[3] Cancer Genome Atlas Network, "Comprehensive genomic characterization of head and neck squamous cell carcinomas," Nature, vol. 517, no. 7536, pp. 576-582, 2015.

[4] A. Panwar, R. Batra, W. M. Lydiatt, and A. K. Ganti, "Human papilloma virus positive oropharyngeal squamous cell carcinoma: a growing epidemic," Cancer Treatment Reviews, vol. 40, no. 2, pp. 215-219, 2014.

[5] H. J. Ryu, E. K. Kim, S. J. Heo, B. C. Cho, H. R. Kim, and S. O. Yoon, "Architectural patterns of p16 immunohistochemical expression associated with cancer immunity and prognosis of head and neck squamous cell carcinoma," APMIS-Acta Pathologica, Microbiologica et Immunologica Scandinavica, vol. 125, no. 11, pp. 974-984, 2017.

[6] E. Gelwan, I. Malm, A. Khararjian, C. Fakhry, J. A. Bishop, and W. H. Westra, "Nonuniform Distribution of High-risk Human Papillomavirus in Squamous Cell Carcinomas of the Oropharynx," The American Journal of Surgical Pathology, vol. 41, no. 12, pp. 1722-1728, 2017.

[7] A. J. Hobbs, N. T. Brockton, T. W. Matthews et al., "Primary treatment for oropharyngeal squamous cell carcinoma in Alberta, Canada: A population-based study," Head \& Neck, vol. 39, no. 11, pp. 2187-2199, 2017.

[8] J. Mallen-St Clair, M. Alani, M. B. Wang, and E. S. Srivastan, "Human papillomavirus in oropharyngeal cancer: The changing face of a disease," Biochimica et Biophysica Acta (BBA) - Reviews on Cancer, vol. 1866, no. 2, pp. 141-150, 2016.

[9] P. R. Aguiar Pastrez, V. S. Mariano, A. M. Costa et al., "The relation of HPV infection and expression of p53 and p16 proteins in esophageal squamous cells carcinoma," Journal of Cancer, vol. 8, no. 6, pp. 1062-1070, 2017.

[10] H. H. Al-Khalaf, S. C. Nallar, D. V. Kalvakolanu, and A. Aboussekhra, "p16INK4A enhances the transcriptional and the apoptotic functions of p53 through DNA-dependent interaction," Molecular Carcinogenesis, vol. 56, no. 7, pp. 1687-1702, 2017.

[11] A. M. Mills, D. C. Dirks, M. D. Poulter, S. E. Mills, and M. H. Stoler, "HR-HPV E6/E7 mRNA in situ hybridization. Validation against PCR, DNA in situ hybridization, and p16 immunohistochemistry in 102 samples of cervical, vulvar, anal, and head and neck neoplasia," The American Journal of Surgical Pathology, vol. 41, no. 5, pp. 607-615, 2017.

[12] K. A. Lang Kuhs, A. R. Kreimer, S. Trivedi et al., "Human papillomavirus 16 E6 antibodies are sensitive for human papillomavirus-driven oropharyngeal cancer and are associated with recurrence," Cancer, vol. 123, no. 22, pp. 4382-4390, 2017.
[13] T. Nakagawa, K. Matsusaka, K. Misawa et al., "Frequent promoter hypermethylation associated with human papillomavirus infection in pharyngeal cancer," Cancer Letters, vol. 407, pp. 2131, 2017.

[14] E.-S. Prigge, C. Toth, G. Dyckhoff et al., "P16INK4a/Ki-67 coexpression specifically identifies transformed cells in the head and neck region," International Journal of Cancer, vol. 136, no. 7, pp. 1589-1599, 2015.

[15] K. K. Ang, J. Harris, R. Wheeler, R. Weber et al., "Human Papillomavirus and Survival of Patients with Oropharyngeal Cancer," The New England Journal of Medicine, vol. 363, no. 1, pp. 24-35, 2010.

[16] S. H. Huang, W. Xu, J. Waldron et al., "Refining American joint committee on cancer/union for international cancer control TNM stage and prognostic groups for human papillomavirusrelated oropharyngeal carcinomas," Journal of Clinical Oncology, vol. 33, no. 8, pp. 836-845, 2015.

[17] K. R. Dahlstrom, A. S. Garden, W. J. N. William, M. Y. Lim, and E. M. Sturgis, "Proposed staging system for patients with hpvrelated oropharyngeal cancer based on nasopharyngeal cancer n categories," Journal of Clinical Oncology, vol. 34, no. 16, pp. 1848-1854, 2016.

[18] A. K. Chaturvedi, W. F. Anderson, J. Lortet-Tieulent et al., "Worldwide trends in incidence rates for oral cavity and oropharyngeal cancers," Journal of Clinical Oncology, vol. 31, no. 36, pp. 4550-4559, 2013.

[19] Z. Wang, R.-H. Xia, D.-X. Ye, and J. Li, "Human papillomavirus 16 infection and TP53 mutation: Two distinct pathogeneses for oropharyngeal squamous cell carcinoma in an eastern Chinese population," PLoS ONE, vol. 11, no. 10, Article ID e0164491, 2016.

[20] E. W. H. Lam, J. Y. W. Chan, A. B. W. Chan et al., "Prevalence, clinicopathological characteristics, and outcome of human papillomavirus-associated oropharyngeal cancer in southern Chinese patients," Cancer Epidemiology, Biomarkers \& Prevention, vol. 25, no. 1, pp. 165-173, 2016.

[21] D. Rischin, R. J. Young, R. Fisher et al., "Prognostic significance of p16INK4Aand human papillomavirus in patients with oropharyngeal cancer treated on TROG 02.02 phase III trial," Journal of Clinical Oncology, vol. 28, no. 27, pp. 4142-4148, 2010.

[22] A. Al-Kaabi, L. W. van Bockel, A. J. Pothen, and S. M. Willems, "P16 ${ }^{I N K 4 A}$ and $\mathrm{p} 14^{A R F}$ gene promoter hypermethylation as prognostic biomarker in oral and oropharyngeal squamous cell carcinoma: a review," Disease Markers, vol. 2014, Article ID 260549, 8 pages, 2014.

[23] P. Molony, N. Kharytaniuk, S. Boyle et al., "Impact of positive margins on outcomes of oropharyngeal squamous cell carcinoma according to p16 status," Head \& Neck, vol. 39, no. 8, pp. 1680-1688, 2017.

[24] F. Wang, H. Zhang, Y. Xue et al., "A systematic investigation of the association between HPV and the clinicopathological parameters and prognosis of oral and oropharyngeal squamous cell carcinomas," Cancer Medicine, vol. 6, no. 5, pp. 910-917, 2017. 


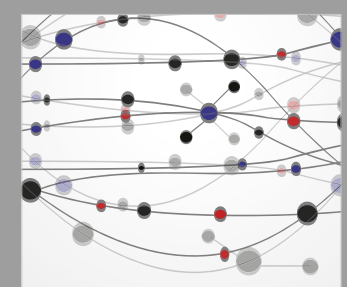

The Scientific World Journal
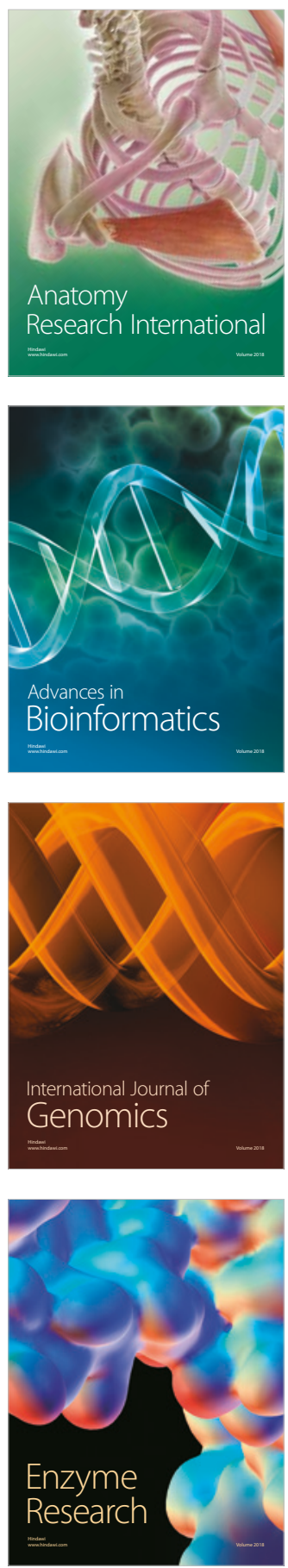
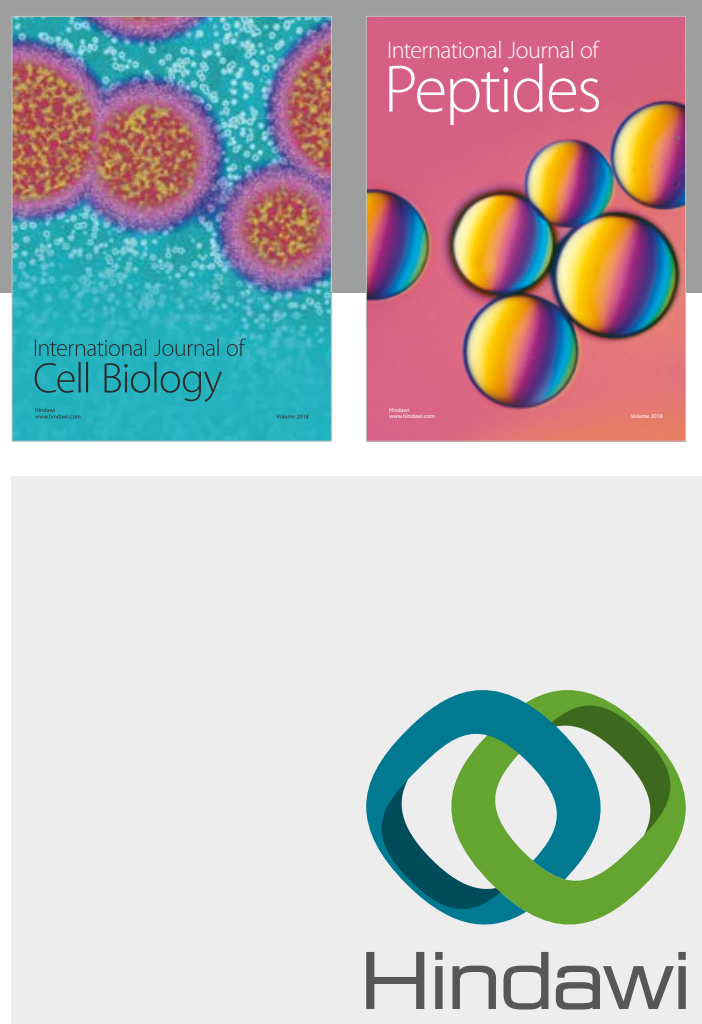

Submit your manuscripts at

www.hindawi.com
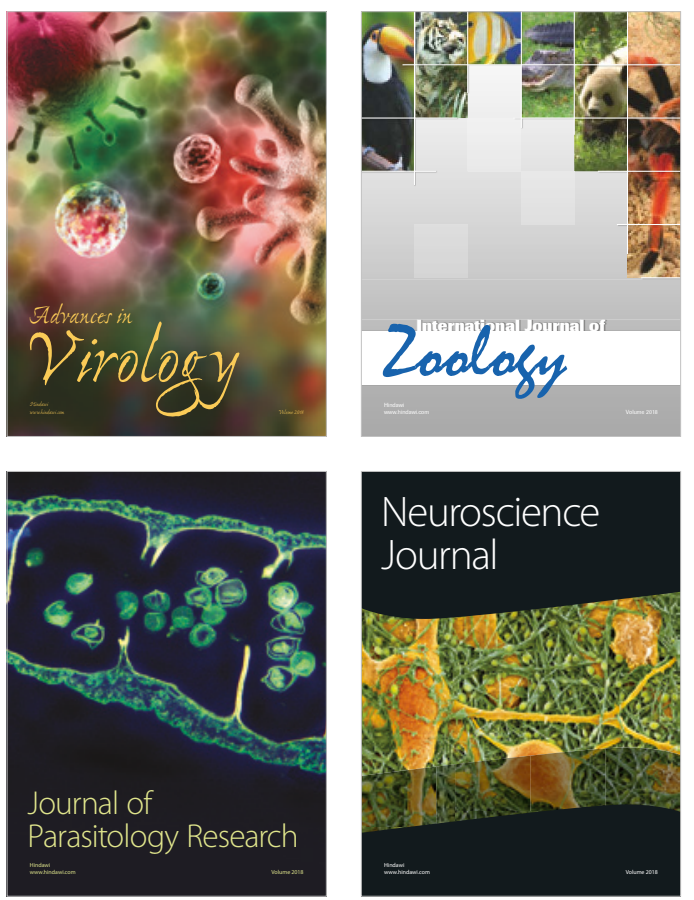
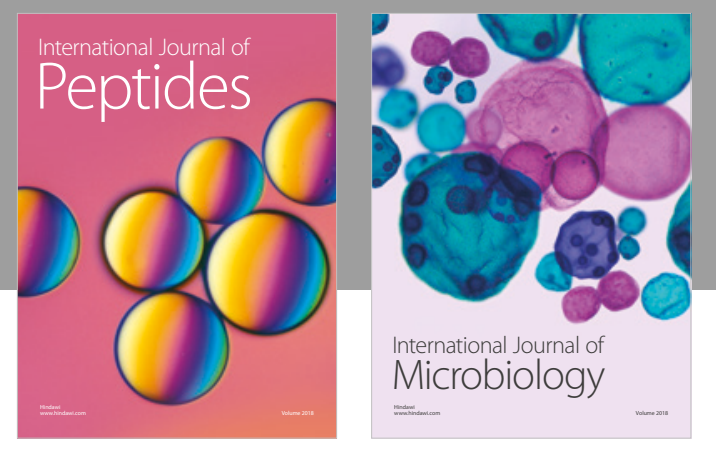

nternational Journal of Microbiology
Journal of
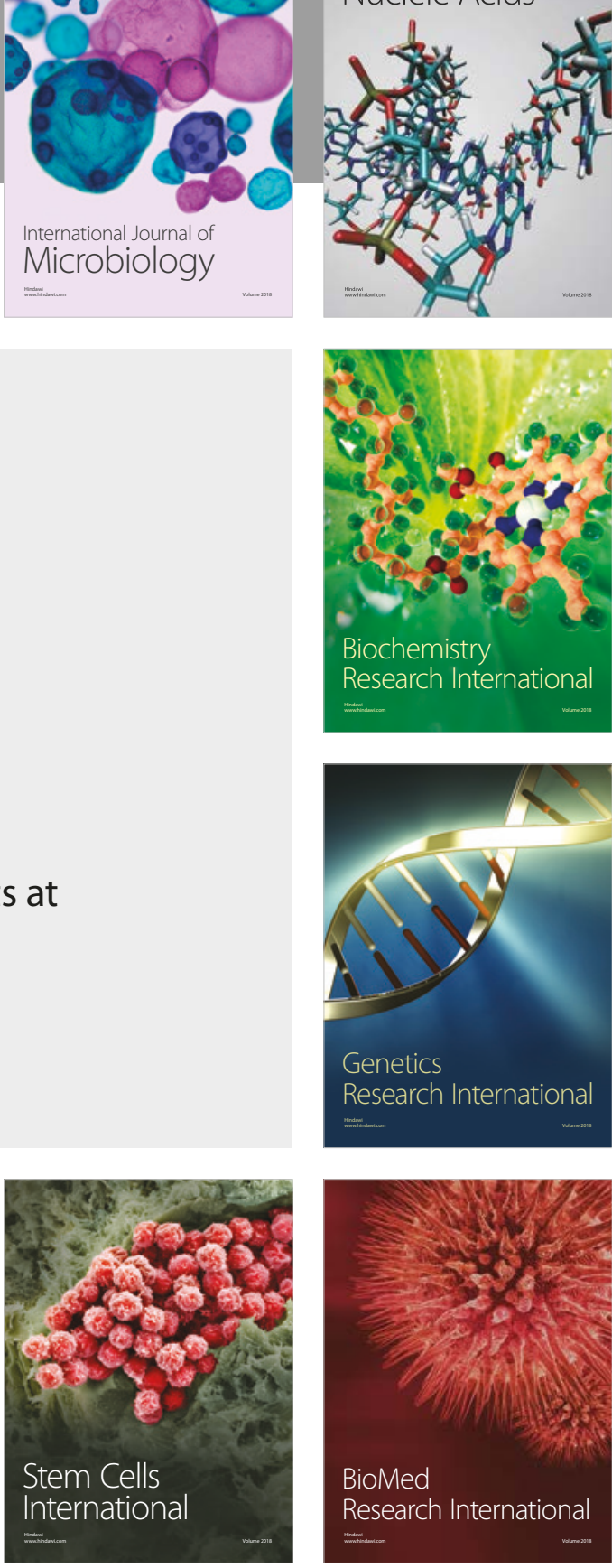
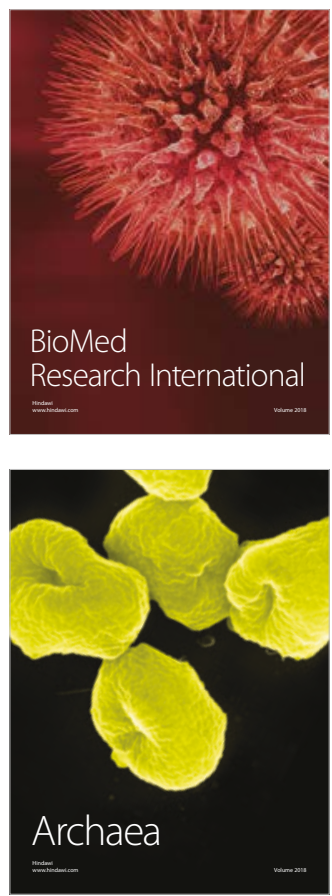